\title{
Slice and Rearrange to Form Quarter-wavelength Plates: Numerical Simulation in One Dimension
}

\author{
Chi-Chang Chang, ${ }^{1}$ Shin-Ku Lee, ${ }^{2 *}$ Mingtsu Ho, ${ }^{3 * *}$ and Chenyu Chen ${ }^{1}$ \\ ${ }^{1}$ Department of Architecture, National Cheng Kung University, \\ No. 1, Dasyue Rd, Tainan City, Taiwan \\ ${ }^{2}$ Research Center for Energy Technology and Strategy, National Cheng Kung University, \\ No. 1, Dasyue Rd, Tainan City, Taiwan \\ ${ }^{3}$ Graduate School of Opto-Mechatronics and Materials, WuFeng University, \\ 117 Jian Kuo Road Sect. 2, Min Shong, Taiwan
}

(Received July 6, 2018; accepted October 18, 2018)

Keywords: method of characteristics, quarter-wavelength, antireflective glass

In this paper, the computational results of numerical simulations for newly proposed structures combining two quarter-wavelength $(\mathrm{QW})$ slabs in one dimension are presented. The new QW plate is composed of alternate layers of two different QW slabs that are made of nonmagnetic dielectric materials $\mathrm{A}$ and $\mathrm{B}$, characterized by the dielectric constants $\varepsilon_{r A}$ and $\varepsilon_{r B}$, which satisfy the relation $\left(\varepsilon_{r A}\right)^{2}=\varepsilon_{r B}>1$, in order to minimize the reflection from the structures. Slabs A and B are, in theory, uniformly sliced into $N+1$ and $N$ pieces, respectively, or vice versa. They are then respectively rearranged into two different structures, $\mathrm{A}(\mathrm{BA})_{N}$ and $\mathrm{B}(\mathrm{AB})_{N}$, which are numerically proved to function as QW plates. Compared with the traditional antireflection coating (ARC) techniques, the newly proposed structures have the advantages that every component of each type of material is identical in thickness and that they are easy to assemble. The idea of the proposed structures is numerically supported by simulation results obtained through the application of the method of characteristics (MOC). The wavelength of interest is set to $550 \mathrm{~nm}$, which corresponds to green light. The numerical results, in both the time and frequency domains, demonstrate that the proposed structures function as antireflective glasses that can be straightforwardly fabricated and used to increase the energy efficiency and reduce the environmental impact of buildings as well as to enhance the performance of some wavelength-sensitive optical sensors. It can also be used as a new coating material for solar panels to single out the specific wavelength of light and hence to protect the solar panel from infrared and ultraviolet radiations.

\section{Introduction}

In this paper, we propose an innovative idea of rearranged structures of two equally sliced quarter-wavelength $(\mathrm{QW})$ slabs, each comprising a stack of two different types of transparent sheets in alternating order. The proposed structures are numerically demonstrated to have the functions of antireflective plates by simulation results obtained through the application of

\footnotetext{
*Corresponding author: e-mail: sklee1015@gmail.com

** Corresponding author: e-mail: homt@wfu.edu.tw

https://doi.org/10.18494/SAM.2019.2109
} 
the method of characteristics (MOC). The advantages of the proposed arrangements over the traditional antireflection coating techniques are that each component of the two materials is uniform in thickness and that they are easy to synthesize.

To regulate and supervise the energy performance of buildings, a variety of building energy rating systems have been established worldwide as tools to help monitor and improve the overall building energy efficiency. Many incentive-based initiatives have also been created to encourage homeowners to select high-performance energy-efficient windows, window manufacturers to develop low-heat-absorption windows, and architects and builders in the design stage to follow energy management standards, particularly for air-conditioned buildings. Therefore, high-quality windows are those that have been designed with the environmental impact and energy efficiency in mind.

The proposed structures were motivated by the fiber Bragg grating (FBG), which is utilized in an optical fiber to reflect certain wavelengths of light and let all others pass through. ${ }^{(1)}$ In the realization of an FBG, a periodic variation in the refractive index is generated in the fiber core, which functions as a wavelength-specific dielectric mirror or wavelength-selective reflector. The proposed combinational QW plates have a different structure from the FBG and feature low reflectivity near the designed wavelength. Interference-based coatings were invented and developed by Alexander Smakula in 1935. ${ }^{(2)}$ One type of optical device used to reduce reflectivity is the antireflective or antireflection coating (ARC), which is typically applied to the surface of lenses or glasses. The rearranged QW plates are relatively simple in terms of both the components and arrangement compared with the known ARC techniques.

In the present study, the MOC is employed as a numerical procedure to solve Maxwell's equations and provide numerical evidence that the proposed structures function as quarterwavelength plates. The MOC was developed for the direct numerical solutions of the timedomain Maxwell's equations and was first reported to produce well-matched results with those generated by the finite-difference time-domain technique in $1995 .^{(3,4)}$ In Ref. 4, a study of electromagnetic (EM) fields scattered from a perfect electric conductor (PEC) strip of infinite length under the illumination of a plane EM pulse with a Gaussian envelope was reported. The MOC has also been applied to the study of the EM reflection from traveling and/or vibrating perfect surfaces and yielded results in good agreement with theoretical values. ${ }^{(5,6)}$ In both cases, MOC was carried out in one dimension in cooperation with the relativistic boundary conditions to include the relativistic effects due to the motion of the object of interest. In the study reported in Ref. 6, the PEC strip traveled at an extremely high velocity near $90 \%$ of the speed of light, while either advancing or retreating. Moreover, MOC was found to generate numerical results demonstrating reasonable trends in the EM problems due to the effects of the conductivity of a medium on the propagation of EM fields into a conducting dielectric halfspace $^{(7)}$ and the effects of a lossless nonuniform dielectric slab on the transmission of EM fields. ${ }^{(8)}$ To overcome the grid distortion problems originating from a rotating cylinder, MOC was used with the passing center swing back grid (PCSBG) technique and the modified O-type grid system and to produce numerical results exhibiting reasonable trends for the EM fields scattered from rotating circular dielectric cylinders, where plane EM pulses with a Gaussian envelope were used as excitation sources. ${ }^{(9-12)}$ 


\section{Governing Equations and Boundary Conditions}

In the present study, MOC is employed for the numerical solution of the time-domain Maxwell equations. In the one-dimensional numerical model, a stack of transparent sheets made of different materials is subjected to the illumination of a plane EM pulse with a Gaussian envelope. Because the present problem does not involve any sources, the governing equations are the following time-domain Maxwell's equations in free space:

$$
\begin{aligned}
& \frac{\partial \vec{B}}{\partial t}+\nabla \times \vec{E}=0, \\
& \frac{\partial \vec{D}}{\partial t}-\nabla \times \vec{H}=0,
\end{aligned}
$$

where $\vec{E}$ and $\vec{H}$ are the electric and magnetic field strengths, and $\vec{D}$ and $\vec{B}$ are the electric and magnetic flux densities, respectively. The electric and magnetic field components satisfy the relations $\vec{D}=\varepsilon_{r} \varepsilon_{o} \vec{E}$ and $\vec{B}=\mu_{r} \mu_{o} \vec{H}$, where $\varepsilon_{o}$ and $\mu_{o}$ are the permittivity and permeability of vacuum, and $\varepsilon_{r}$ and $\mu_{r}$ are those of the medium through which the EM fields propagate, respectively. For the current simulation, because the EM fields are normally incident on a planar boundary, both $\vec{E}$ and $\vec{H}$ are parallel to the surface of the medium layers, and because no field components exist along the direction of propagation, Maxwell's equations can be rewritten in the simplified version shown below. In the absence of any sources and provided that the incident EM fields propagate in the positive- $x$ direction, the incident electric fields are polarized along the $z$-axis $\left(\vec{E}=\hat{z} E_{z}\right)$ and the incident magnetic fields are thus in the negative- $y$ direction $\left(\vec{B}=-\hat{y} B_{y}\right)$.

The numerical procedure of the MOC starts with expressing Maxwell's equations in the form of the Euler equation, then transforming them from the Cartesian system $(t, x)$ to the curvilinear system $(\tau, \xi)$. Maxwell's equations become

$$
\frac{\partial Q}{\partial \tau}+\frac{\partial F}{\partial \xi}=0
$$

where

$$
\begin{gathered}
Q=J q, \\
F=J \xi_{x} f, \\
q=\left[\begin{array}{l}
B_{y} \\
D_{z}
\end{array}\right],
\end{gathered}
$$




$$
f=\left[\begin{array}{l}
-E_{z} \\
-H_{y}
\end{array}\right],
$$

and the symbol $J$ is the Jacobian of the inverse transformation and defined in terms of the finite difference as

$$
J=\left|\frac{\Delta x}{\Delta \xi}\right|
$$

Applying the central difference operator on the variable $F$ in Eq. (3), one obtains

$$
\delta_{k}(F)=(F)_{k+\frac{1}{2}}-(F)_{k-\frac{1}{2}},
$$

where the cell index $k$ designates the grid center and the values of $\pm 1 / 2$ indicate the two interfaces of the cell. Equation (9) is interpreted as the change in $F$ in the $k$ th cell being the difference in flux between the two interfaces. Note that the MOC evaluates the flux at every grid boundary and then solves for the difference in flux in every cell. With the application of the central difference operator and finite difference operator, Eq. (3) becomes

$$
\frac{Q^{n+1}-Q^{n}}{\Delta \tau}+\frac{\delta_{i} F}{\Delta \xi}=0 .
$$

Note that the finite difference operator is applied to the first denominator of Eq. (10) in time and the second denominator in space. Consequently, the first term is the forward-difference approximation of the temporal derivative of $Q$ associated with two superscripts $(n+1)$ and $(n)$, which are two successive numerical time steps. The second term is the approximation of the spatial derivative of $F$ for the $i$ th cell, where the numerator is the difference in flux between the two cell walls. The flux vector splitting technique and the lower-upper approximate factorization scheme are then used in collaboration with the MOC to solve the system of equations. ${ }^{(13,14)}$

In the numerical model, a plane EM pulse with a Gaussian envelope is set to normally propagate onto a stack of transparent sheets that have been numerically proved to function as a QW antireflection device. Since the EM fields propagate into and out of clear sheets made of two different materials, there are three types of boundary: air-dielectric, dielectric-air, and dielectric-dielectric. The boundary conditions are that the tangential components of both the electric and magnetic field strengths must be continuous across the boundary, i.e., $E_{z 1}=E_{z 2}$ and $H_{y 1}=H_{y 2}$.

At the outermost truncated numerical boundaries, appropriate boundary conditions guarantee that the outward-bound EM fields continue propagating outward. 


\section{Proposed Structures and Definition of Problem}

With the proposition that the two transparent slabs are made of two different nonmagnetic $\left(\mu_{r}=1\right)$ materials A and B respectively specified by the dielectric constants $\varepsilon_{r A}$ and $\varepsilon_{r B}$, there are two different arrangements of the proposed $\mathrm{QW}$ antireflection structures: $\mathrm{A}(\mathrm{BA})_{N}$ and $\mathrm{B}(\mathrm{AB})_{N}$. Depicted in Fig. 1(c) is a schematic diagram of the $\mathrm{A}(\mathrm{BA})_{N}$ structure, which is made from the two QW plates with thicknesses of $\lambda_{A} / 4$ and $\lambda_{B} / 4$ shown in Figs. 1(a) and 1(b), respectively. In theory, slabs $\mathrm{A}$ and $\mathrm{B}$ are uniformly sliced into $N+1$ and $N$ pieces. $\lambda_{A}$ and $\lambda_{B}$ are the two wavelengths inside the two different materials corresponding to a wavelength of $\lambda_{o}$ in free space. The thicknesses of the sliced sheets are defined as

$$
\begin{gathered}
d_{A}=\frac{\lambda_{o}}{4(N+1) \sqrt{\varepsilon_{r A}}}, \\
d_{B}=\frac{\lambda_{o}}{4(N) \sqrt{\varepsilon_{r B}}} .
\end{gathered}
$$

Note that $N$ is a positive integer. For example, if $N$ equals 10 , then the total number of sheets of the rearranged structure is 21 . For the $\mathrm{A}(\mathrm{BA})_{N}$ arrangement, there is always one $\varepsilon_{r B}$ sheet between two $\varepsilon_{r A}$ sheets. The other proposed structure, $\mathrm{B}(\mathrm{AB})_{N}$, is formed by slicing slabs $\mathrm{A}$ and B into $N$ and $N+1$, respectively, and rearranging them into a stack of transparent sheets such that every $\varepsilon_{r A}$ sheet is sandwiched by two $\varepsilon_{r B}$ sheets.

In Fig. 2, the $z$-polarized incident electric field strength has a Gaussian profile in time with pulse width $T$. The time interval of $2 T$, which is measured at the level of $\exp (-0.5)$ across the Gaussian pulse, is labeled in the diagram. The maximum electric field strength of the incident EM pulse is set to $1 \mathrm{~V} / \mathrm{m}$.

The incident plane EM pulse initially propagates in the positive- $x$ direction toward the rearranged structure. To observe the antireflective effect at the designed wavelength of $550 \mathrm{~nm}$,

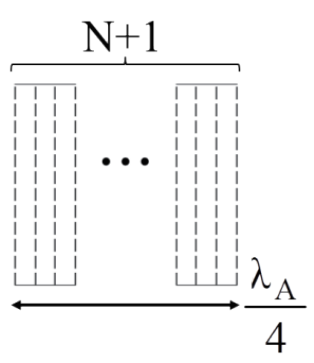

(a)

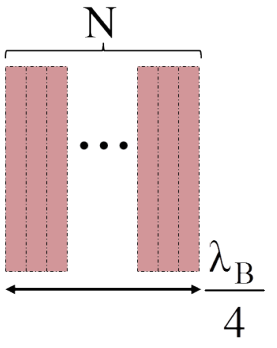

(b)

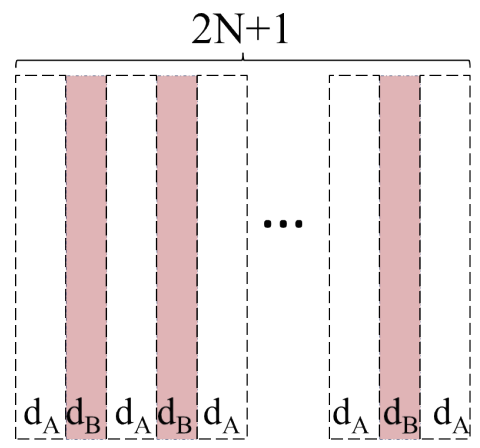

(c)

Fig. 1. (Color online) Two QW slabs and the proposed $\mathrm{A}(\mathrm{BA})_{N}$ structure. 


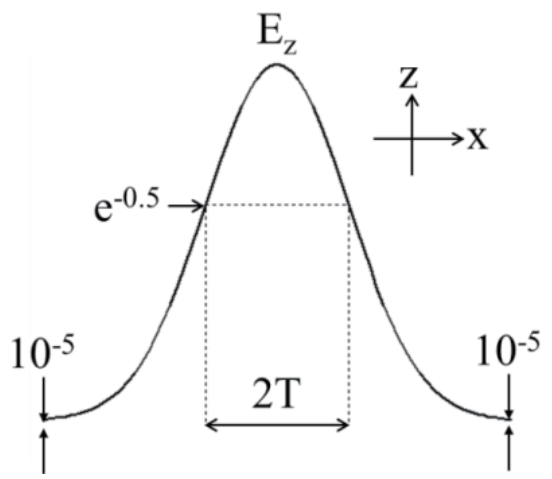

Fig. 2. Incident Gaussian pulse.

the pulse width $T$ of the incident Gaussian EM pulse, set to about $7 \times 10^{-19} \mathrm{~s}$, is truncated at both ends with a cutoff level of $10^{-5}$ and consequently, has a spatial span of $200 \mathrm{~nm}$ between the two cutoff points. The corresponding spectrum of the incident pulse has a maximum content of about $10^{16} \mathrm{~Hz}$ and a shortest wavelength of about $27 \mathrm{~nm}$.

The transparent slabs used in the proposed structure are made of two types of nonmagnetic materials $\mathrm{A}$ and $\mathrm{B}$ with dielectric constants $\varepsilon_{r A}$ and $\varepsilon_{r B}$, respectively. According to Ref. 15, to maximize the transmission across the interface between the two media, $\varepsilon_{r A}$ and $\varepsilon_{r B}$ should be chosen to satisfy the relation

$$
\left(\varepsilon_{r A}\right)^{2}=\varepsilon_{r B}>1
$$

The idea of the proposed structures is firstly to uniformly slice the above-mentioned QW slabs into $N$ and $N+1$ pieces and then to rearrange them to form another QW slab. The numerical discretization usually sets a limit on the accuracy of the parameters, which always must be integers. For instance, the thickness of every sliced sheet used in the numerical model must be represented by an integer. When the actual thickness is not attainable, deviation of the resultant wavelength from the designed wavelength will occur. For a grid density of 10 points per nanometer, $\varepsilon_{r A}=1.5, \varepsilon_{r B}=2.25=\left(\varepsilon_{r A}\right)^{2}$, and $\lambda_{o}=550 \mathrm{~nm}$. Summarized in Tables 1 and 2 are two sets of numbers used in the numerical model. Each table includes four cases: $N=5,10$, 15 , and 20 .

Some of the numbers listed in Tables 1 and 2 are as close approximations as possible to $d_{A}$ and $d_{B}$. Note that $\lambda_{A}^{\prime}, \lambda_{B}^{\prime}$, and $\lambda_{o}^{\prime}$ are the numerical wavelengths inside the media and free space, demonstrating how the approximated values deviate from the actual values $\left(\lambda_{A}, \lambda_{B}\right.$, and $\lambda_{o}$ ) and that the discretization of space results in these deviations since a fractional number of grid points is not acceptable. On the basis of the numbers given in Tables 1 and 2, the total thicknesses of the proposed structures are listed in Table 3. Compared with the theoretical value, $\mathrm{B}(\mathrm{AB})_{N}$ arrangements have larger deviations than $\mathrm{A}(\mathrm{BA})_{N}$ arrangements. 
Table 1

Numbers used for the proposed $\mathrm{A}(\mathrm{BA})_{N}$ structure $\left(\lambda_{O}=550 \mathrm{~nm}\right)$.

\begin{tabular}{rcccccc}
\hline \multirow{2}{*}{$N$} & \multicolumn{3}{c}{$\varepsilon_{r A}=1.5 ; \lambda_{A}=449.07 \mathrm{~nm}$} & \multicolumn{3}{c}{$\varepsilon_{r B}=2.25 ; \lambda_{B}=366.67 \mathrm{~nm}$} \\
\cline { 2 - 7 } & $d_{A}(\mathrm{~nm})$ & $\lambda_{A}^{\prime}(\mathrm{nm})$ & $\lambda_{O A}^{\prime}(\mathrm{nm})$ & $d_{B}(\mathrm{~nm})$ & $\lambda_{B}^{\prime}(\mathrm{nm})$ & $\lambda_{o B}^{\prime}(\mathrm{nm})$ \\
\hline 5 & 18.70 & 448.80 & 549.67 & 18.30 & 366.00 & 549.00 \\
10 & 10.20 & 448.80 & 549.67 & 9.20 & 368.00 & 552.00 \\
15 & 7.00 & 448.00 & 548.69 & 6.10 & 366.00 & 549.00 \\
20 & 5.40 & 453.60 & 555.54 & 4.60 & 368.00 & 552.00 \\
\hline
\end{tabular}

Table 2

Numbers used for the proposed $\mathrm{B}(\mathrm{AB})_{N}$ structure $\left(\lambda_{o}=550 \mathrm{~nm}\right)$.

\begin{tabular}{rcccccc}
\hline \multirow{2}{*}{$N$} & \multicolumn{3}{c}{$\varepsilon_{r B}=2.25 ; \lambda_{B}=366.67 \mathrm{~nm}$} & \multicolumn{3}{c}{$\varepsilon_{r A}=1.5 ; \lambda_{A}=449.07 \mathrm{~nm}$} \\
\cline { 2 - 7 } & $d_{B}(\mathrm{~nm})$ & $\lambda_{B}^{\prime}(\mathrm{nm})$ & $\lambda_{O B}^{\prime}(\mathrm{nm})$ & $d_{A}(\mathrm{~nm})$ & $\lambda_{A}^{\prime}(\mathrm{nm})$ & $\lambda_{O A}^{\prime}(\mathrm{nm})$ \\
\hline 5 & 15.30 & 367.20 & 550.80 & 22.40 & 448.00 & 548.80 \\
10 & 8.30 & 365.20 & 547.80 & 11.20 & 448.00 & 548.80 \\
15 & 5.70 & 364.80 & 547.20 & 7.50 & 450.00 & 551.25 \\
20 & 4.40 & 369.60 & 554.40 & 5.60 & 448.00 & 548.80 \\
\hline
\end{tabular}

Table 3

Total thickness of proposed structures in $\mathrm{nm}$.

\begin{tabular}{lcccc}
\hline$N$ & 5 & 10 & 15 & 20 \\
\hline $\mathrm{A}(\mathrm{BA})_{N}$ & 203.7 & 204.2 & 203.5 & 205.4 \\
$\mathrm{~B}(\mathrm{AB})_{N}$ & 210.9 & 206.2 & 205.5 & 205.6 \\
\hline
\end{tabular}

Theoretical value $=203.9 \mathrm{~nm}$.

\section{Numerical Results}

When EM fields propagate onto a stack of transparent dielectric sheets, multiple reflections and multiple transmissions are expected to occur. Figures 3 and 4 show series of electric fields at several time instances for the $\mathrm{A}(\mathrm{BA})_{N}$ and $\mathrm{B}(\mathrm{AB})_{N}$ structures, respectively, where the times are intervals of $100 \mathrm{~nm} / \mathrm{c}$ and $\mathrm{c}$ is the speed of light. The front edge of the proposed structure is located at $x=200 \mathrm{~nm}$ and the dotted lines represent the components of the proposed structures. From Figs. 3 and 4 , at $t=0$, the initial electric field strength is $1 \mathrm{~V} / \mathrm{m}$, only electric fields having magnitudes greater than $5 \mathrm{mV} / \mathrm{m}$ are shown, the EM fields exhibit multiple reflections and multiple transmissions for $N=5$ [Figs. 3(a) and 4(a)], and there is only one detectable reflected waveform for $N=20$ in both the $\mathrm{A}(\mathrm{BA})_{N}$ and $\mathrm{B}(\mathrm{AB})_{N}$ structures [Figs. 3(b) and 4(b)].

More numerical results providing evidence that the proposed structures can act as antireflective plates are shown in Fig. 5, where the reflected electric fields recorded at $100 \mathrm{~nm}$ in front of the structures are plotted as functions of time. Closer observation of the primary reversed reflection reveals that the $N=5$ case has the smallest magnitude of the reflected electric field for the $\mathrm{A}(\mathrm{BA})_{N}$ arrangement. On the other hand, for the primary reflection of the $\mathrm{B}(\mathrm{AB})_{N}$ structures, the $N=5$ case has the largest magnitude because the leading sheet is thicker and has a greater dielectric constant. It is also observed that as $N$ increases, the magnitude of the reflection/transmission ripples between the primary and secondary reflections decreases with less reflection from the second edge. There are almost no detectable reflections between the two peaks, especially after the secondary reflection, for the $N=20$ case. 


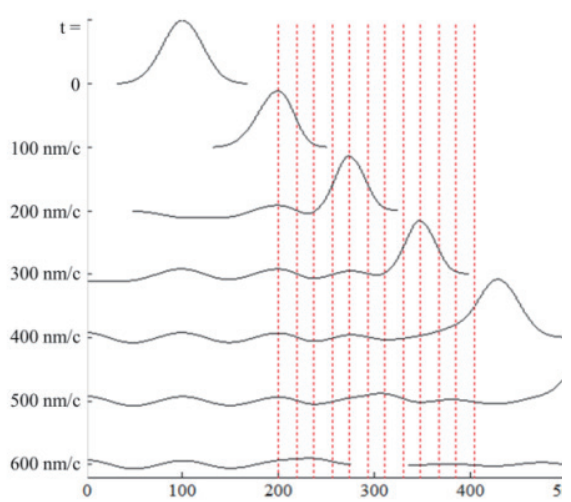

(a)

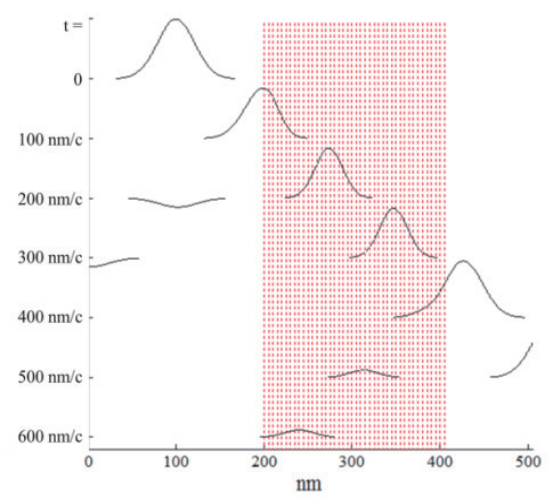

(b)

Fig. 3. (Color online) EM fields propagating onto $\mathrm{A}(\mathrm{BA})_{N}$ structures: (a) $N=5$ and (b) $N=20$.

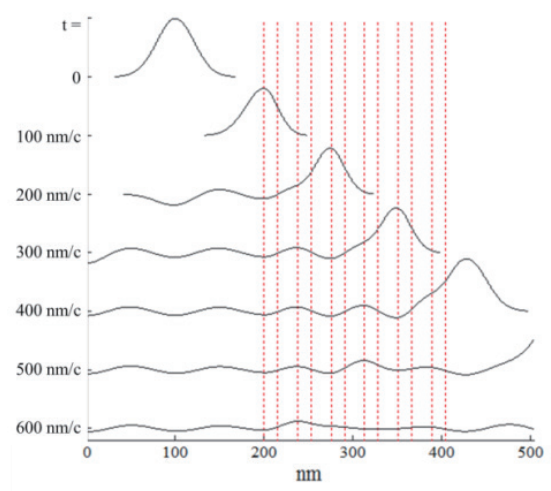

(a)

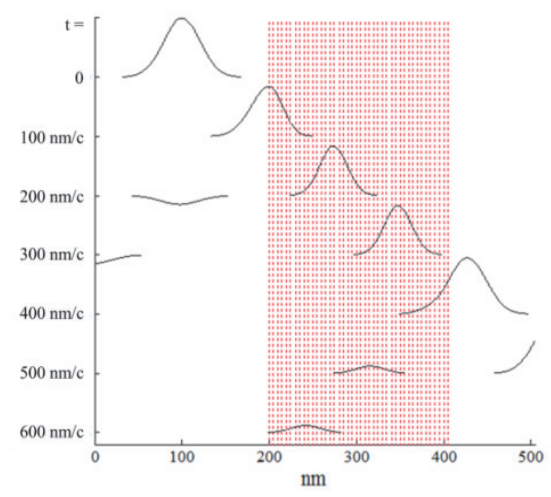

(b)

Fig. 4. (Color online) EM fields propagating onto $\mathrm{B}(\mathrm{AB})_{N}$ structures: (a) $N=5$ and (b) $N=20$.

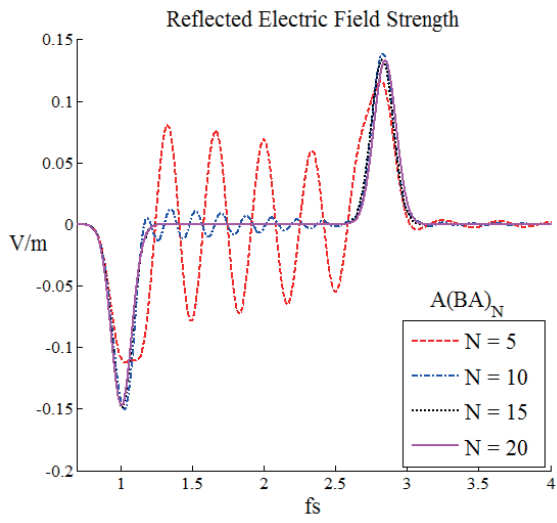

(a)

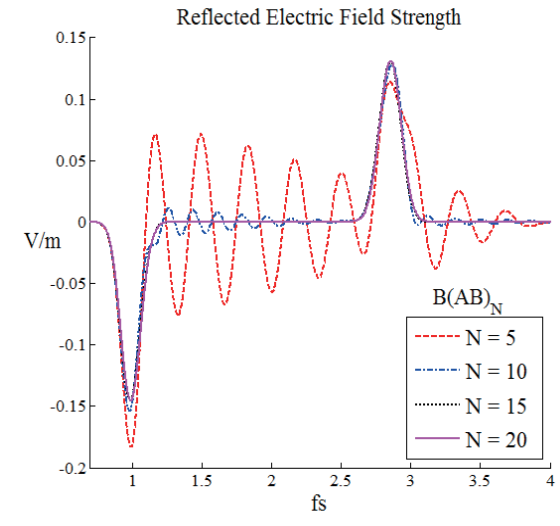

(b)

Fig. 5. (Color online) Reflected electric fields: (a) $\mathrm{A}(\mathrm{BA})_{N}$ and (b) $\mathrm{B}(\mathrm{AB})_{N}$.

Because in Fig. 5 most primary and secondary peaks are not distinguishable, four zoom-in plots are given in Fig. 6. Focusing on these curves, one finds that the results for $N=15$ and 20 almost coincide, as shown in Figs. 6(a) and 6(c). The times of both the primary and secondary peaks are measured and are listed in Tables 4 and 5. 


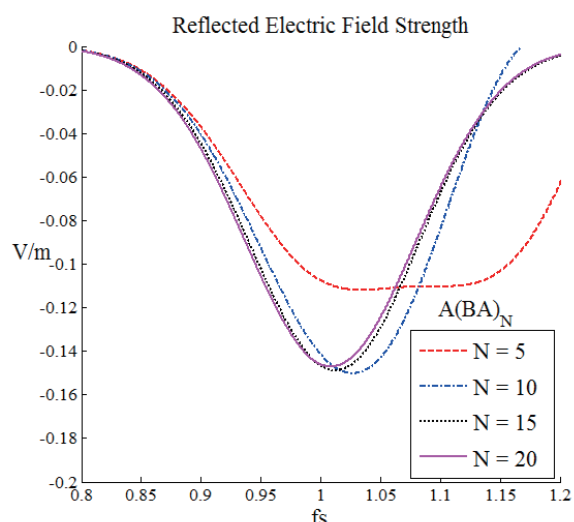

(a)

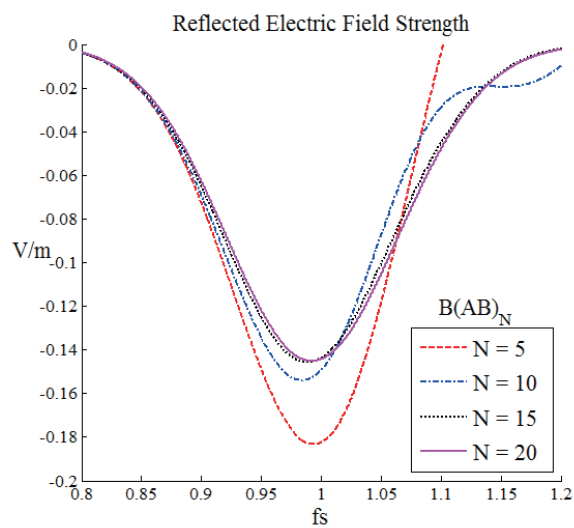

(c)

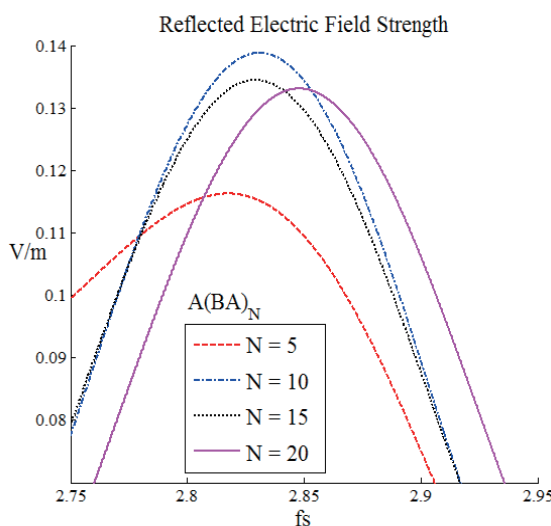

(b)

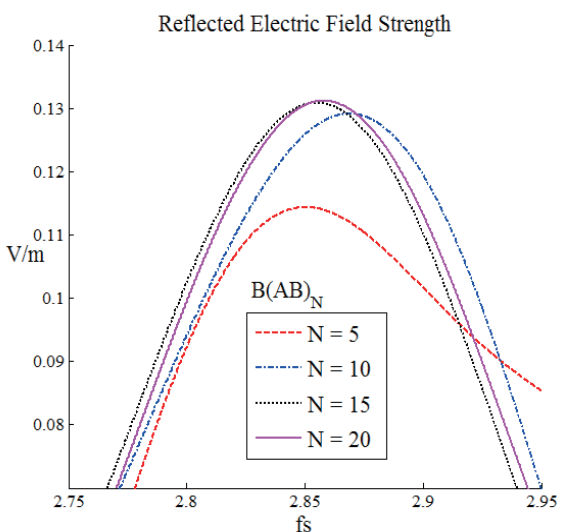

(d)

Fig. 6. (Color online) Zoom-in views of the reflected electric fields: (a), (b) $\mathrm{A}(\mathrm{BA})_{N}$; (c), (d) $\mathrm{B}(\mathrm{AB})_{N}$.

Table 4

Times of the reflection peaks: $\mathrm{A}(\mathrm{BA})_{N}$ structures.

\begin{tabular}{lcccc}
\hline$N$ & 5 & 10 & 15 & 20 \\
\hline Primary (fs) & 1.0350 & 1.0269 & 1.0125 & 1.0085 \\
Secondary (fs) & 2.8175 & 2.8310 & 2.8293 & 2.8481 \\
\hline
\end{tabular}

Table 5

Times of the reflection peaks: $\mathrm{B}(\mathrm{AB})_{N}$ structures.

\begin{tabular}{lcccc}
\hline$N$ & 5 & 10 & 15 & 20 \\
\hline Primary (fs) & 0.9937 & 0.9841 & 0.9885 & 0.9923 \\
Secondary (fs) & 2.8504 & 2.8689 & 2.8552 & 2.8581 \\
\hline
\end{tabular}

The time difference between these two peaks is the time required for the EM fields to travel one round trip inside the structure, which is twice the total distance given in Table 3. The average velocity of the EM fields inside the structure is computed and from it, the equivalent dielectric constant is obtained. Table 6 summarizes the calculated equivalent dielectric constants. Note that the calculated results lie between $\varepsilon_{r A}$ and $\varepsilon_{r B}$ and that the geometric mean value of $\varepsilon_{r A}$ and $\varepsilon_{r B}$ is about 1.84 . 
Further numerical results are provided as evidence that the proposed $\mathrm{A}(\mathrm{BA})_{N}$ and $\mathrm{B}(\mathrm{AB})_{N}$ structures have minimum reflections near the designed wavelength. They are the corresponding spectra (expressed in $\mathrm{nm}$ ) of the reflected electric fields, as shown in Figs. 7 and 8. Although the spectra are too close to be distinguished, minima can be clearly observed in the vicinity of $550 \mathrm{~nm}$. Two zoom-in views of these minima at around $550 \mathrm{~nm}$ are given in Figs. 7(b) and 8(b), from which the computational results of the computed locations of the minimum reflections are summarized in Table 7. It can be clearly seen that the $\mathrm{A}(\mathrm{BA})_{N}$ structures yield better results than the $\mathrm{B}(\mathrm{AB})_{N}$ structures.

Table 6

Calculated equivalent dielectric constants of the proposed structure.

\begin{tabular}{lcccc}
\hline$N$ & 5 & 10 & 15 & 20 \\
\hline $\mathrm{A}(\mathrm{BA})_{N}$ & 1.72 & 1.76 & 1.79 & 1.80 \\
$\mathrm{~B}(\mathrm{AB})_{N}$ & 1.74 & 1.88 & 1.86 & 1.85 \\
\hline
\end{tabular}

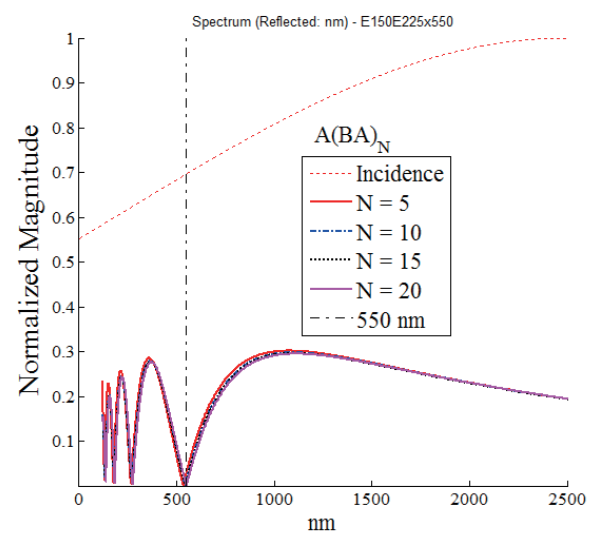

(a)

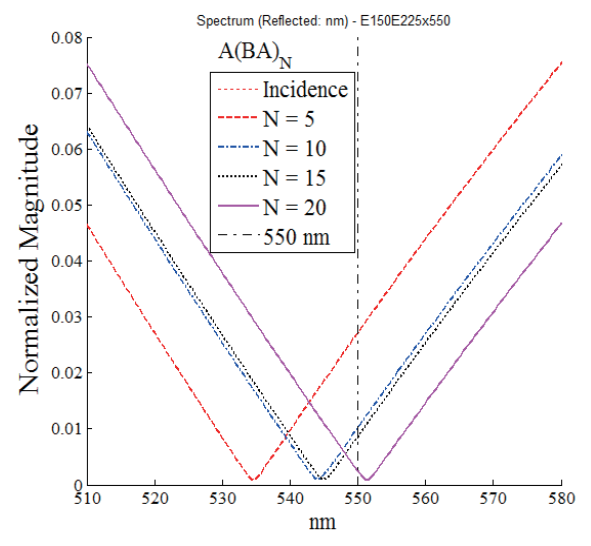

(b)

Fig. 7. (Color online) Spectra of $\mathrm{A}(\mathrm{BA})_{N}$ structures.

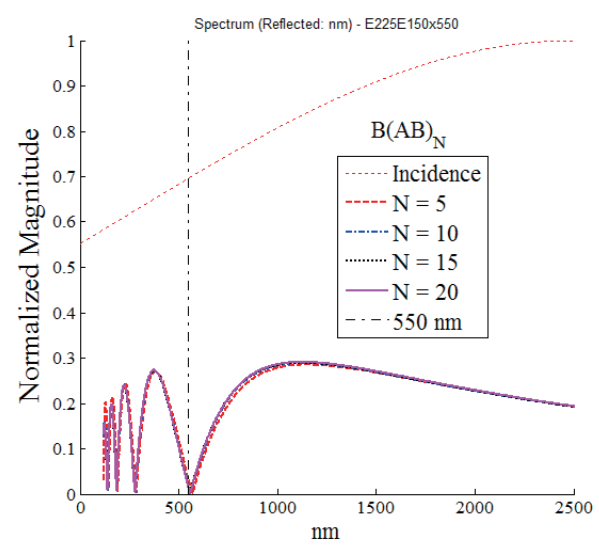

(a)

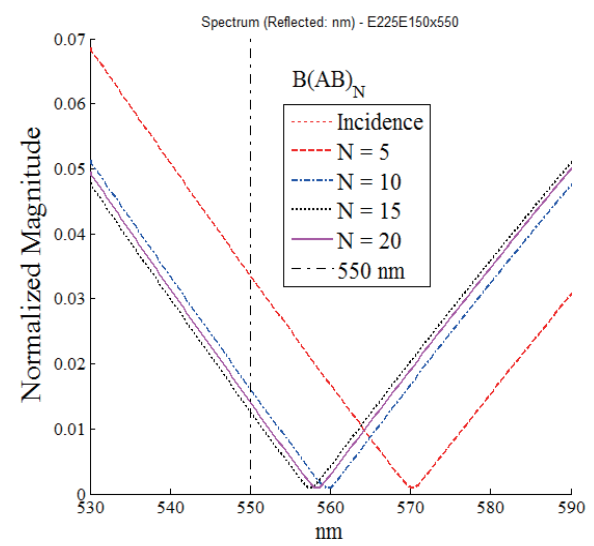

(b)

Fig. 8. (Color online) Spectra of $\mathrm{B}(\mathrm{AB})_{N}$ structures. 
Table 7

Locations of minimum reflections.

\begin{tabular}{lcccc}
\hline$N$ & 5 & 10 & 15 & 20 \\
\hline $\mathrm{A}(\mathrm{BA})_{N}$ & 534.6 & 544.2 & 545.1 & 551.4 \\
$\mathrm{~B}(\mathrm{AB})_{N}$ & 570.4 & 559.9 & 557.8 & 558.2
\end{tabular}

Design wavelength $=550 \mathrm{~nm}$.

\section{Conclusions}

The newly proposed structures have been numerically proved to function as quarterwavelength plates. The advantages of the sliced-and-rearranged QW plates over the traditional antireflective coating techniques are as follows: only two transparent dielectric materials are needed, each material is uniform in thickness, and they are easy to synthesize. Computational results, obtained through the application of the method of characteristics in both the time and frequency domains, showed that the proposed structures have minimum reflectivity near the designed wavelength, where the deviations from the designed wavelength are caused by the discretization process. The equivalent dielectric constants are also calculated and found to lie between those of the two materials. A future task is to carry out numerical simulations for different structures and more complicated configurations.

\section{Acknowledgments}

This research was financially supported by the Ministry of Science and Technology of Taiwan under project MOST 106-3113-E-006-006-CC2. The authors are grateful to the Ministry of Science and Technology of Taiwan.

\section{References}

1 K. O. Hill, Y. Fujii, D. C. Johnson, and B. S. Kawasaki: Appl. Phys. Lett. 32 (1978) 647.

2 H. A. MacLeod: Thin Film Optical Filters (CRC Press, Taylor \& Francis Group, 2010) 4th ed.

3 A. Taflove: Computational Electrodynamics, The Finite-Difference Time-Domain Method (Artech House, Boston, 1995).

4 J. P. Donohoe, J. H. Beggs, and M. Ho: 27th IEEE Southeastern Symp. System Theory (1995).

5 M. Ho: IEEE Trans. Antennas Propag. 54 (2006) 152.

6 M. Ho: J. Appl. Sci. Eng. 17 (2014) 429.

7 M. Ho and F. S. Lai: JEMWA 21 (2007) 1773.

8 M. Ho, F. S. Lai, S. W. Tan, and P. W. Chen: PIER 81 (2008) 197.

9 M. Ho: JEMWA 23 (2009) 389.

10 M. Ho: PIERS Proc. (2009) 1646-1651.

11 M. Ho: PIER 92 (2009) 79-90.

12 M. Ho, L. A. Tsai, and C. J. Tsai: PIER M 45 (2016) 1-8.

13 D. L. Whitfield and M. Janus: AIAA Paper No. 84-1552 (1984).

14 W. Briley, S. Neerarambam, and D. Whitfield: 12th Computational Fluid Dynamics Conf., Fluid Dynamics and Co-located Conferences, A95-36593 (1995).

15 J. Krepelka: Jemná Mechanika A Optika 3 (1992) 53. 